\title{
Supporting Work Activities in Healthcare by Mobile Electronic Patient Records
}

\author{
Jesper Kjeldskov and Mikael B. Skov \\ Aalborg University \\ Department of Computer Science \\ DK-9220 Aalborg East, Denmark \\ \{jesper, dubois\}@cs.auc.dk
}

\begin{abstract}
Supporting work activities in healthcare is highly complex and challenging. This paper outlines the findings from a usability study of a commercial PC based electronic patient record (EPR) system at a large Danish hospital and presents our experiences with the design of a mobile counterpart. First, a number of challenges in relation to the use of traditional desktop-based EPR systems in healthcare were identified. Secondly, a mobile context-aware prototype was designed and implemented, which automatically keeps track of contextual factors such as the physical location of patients and staff, upcoming appointments etc. The usability of the mobile EPR prototype was evaluated in a laboratory as well as in relation to carrying out real work activities at the hospital. Our results indicate that mobile EPR systems can support work activities in healthcare, but that interaction design of such systems must be carefully thought out and evaluated. Specifically, our findings challenge the view of context-awareness being a universally useful paradigm for mobile HCI.
\end{abstract}

\section{Introduction}

An electronic patient record (EPR) is a collection of information about a single patient's history in a hospital. The hospital personnel use the record to diagnose diseases, and to document and coordinate treatment. Within the last 20 years, a considerable amount of effort has been devoted to the development of electronic patient record systems. The primary motivation for this effort is that unlike paper-based patient records, electronic patient records will be accessible to all relevant persons independent of time and location.

The design of electronic patient records is a huge challenge for the HCI community, raising a wide range of still unanswered questions related to issues such as screen layout, interaction design, and integration into work processes. Where should the systems be located and who should enter the data? How do we make sure that input is complete and accurate? How are the different work processes in healthcare structured and coordinated? What is the most useful way of displaying and accessing the vast quantity of patient data? [4]. In the light of these questions, a lot of research has been published in the HCI literature about EPR systems and how to meet challenges related to design and use of computer system in healthcare. Specifically, much 
attention has been given to issues such as information sharing [5], support for cooperation [6] and privacy [12]. While much of this research is based on studies on the use of traditional paper-based patient records, suggesting viable electronic counterparts, however, little research has been published based on studies that inquire into the use of the mass of EPR systems already in use.

In Denmark, there is currently an extensive focus on electronic patient records. The government has decided that by 2005 all Danish hospitals must have replaced the traditional paper-based patient records with electronic ones. However, it is up the regional authorities to decide on the details of deployment. Thus a number of pilot projects are currently in progress with the aim of developing and evaluating electronic patient record systems (see e.g. [2]). In relation to a regional Danish research program entitled "The Digital Hospital" we have studied the use of a commercial EPR system currently in use at a large hospital (IBM IPJ 2.3). In addition to this, an experimental mobile EPR prototype extending the current system's functionality was designed and evaluated. Driving this study, we were concerned with the following research questions: 1) what challenges characterize the use of contemporary electronic patient record systems? 2) How can these challenges be met by improved interaction design?

This paper outlines the findings from the usability evaluation of IBM's PC based EPR system IPJ 2.3 and presents our preliminary experiences with the usability of a mobile counterpart. The paper is structured in the following way. First, we present our initiating usability evaluation. The findings from this evaluation are then outlined as a number of themes describing problems and advantages encountered during use. Subsequently, we present the design of the experimental mobile EPR prototype system, and present the results of a field- and lab-based evaluation of its usability. Finally, we discuss the findings from the usability evaluations of the mobile EPR prototype in the light of the themes identified in the evaluation of the stationary EPR system.

\section{Electronic Patient Record Usability}

We conducted a usability evaluation of IBM's electronic patient record system IPJ 2.3 currently in use at the Hospital of Frederikshavn, Denmark. The evaluation was carried out in a dedicated usability laboratory at the University of Aalborg over a period of two days in May 2002. The evaluation involved two elements: 1) a thinkaloud evaluation with the purpose of identifying usability problems in the electronic patient record system and 2) a series of interviews with the purpose of gaining insight into the integration of the electronic patient record into the work of the nurses.

Preparation: Prior to the evaluation, the research team inquired thoroughly into the work activities at the hospital related to the use of patient records. This was done through observation at the hospital and interviews with key personnel. Also, the research team investigated into the functionality of the electronic patient record system to be evaluated. Based on this, scenarios of use of the system and a number of tasks for the evaluation were produced in collaboration with personnel at the hospital.

Test subjects: The evaluation involved eight trained nurses from the Hospital of Frederikshavn. All nurses were women, aged between 31 and 54 years of age with 
professional work experience ranging from 2 to 31 years. All nurses had attended a course on the IPJ 2.3 system prior to the test amounting to between 14 and 30 hours but none of them had used the system in their daily work. They characterized themselves as novices or beginners in relation to the use of IT in general.

Tasks: The purpose of the usability evaluation was to investigate how the EPR system supports typical work activities for nurses at the hospital. Based on our scenarios, we designed three tasks that were centered on the core purpose of the system such as retrieving information about patients, registering information about treatments, making treatment notes, and entering measurements.

Procedure: The test was based on the think-aloud protocol [13], [11]. The interviews were semi-structured, based on a list of questions and a number of issues that could be raised. Evaluations and interviews were conducted over two days. Each nurse first used the system to solve the tasks. After this, four randomly selected nurses were interviewed about their work and their opinions about the system as well as its integration in and influence on their work.

Test setting: All evaluations and interviews were conducted in a dedicated usability laboratory. A test monitor observed the test subjects, encouraged them to think aloud and asked questions for clarification. Two additional researchers operated the video equipment and took notes in a separate control room. The computer used was a standard PC with a 19" screen and a standard mouse and keyboard matching the hardware used on the hospital.

Data Collection: All evaluation sessions were recorded on digital video. Two remotely controlled motorized cameras captured overall and close-up views of the test subject and test monitor. The image on the PC screen was converted to composite video and mixed with the camera images to one composite video signal.

Data analysis: Following the evaluations a log file was produced from each test. Each of the three researchers then used the video recordings and these log files as empirical foundation for producing three individual lists of usability problems. These lists were then merged to one. The severity of the identified problems was rated as critical, serious or cosmetic based on the guidelines proposed by Molich [9]. The rating was done individually and followed by negotiation in cases of disagreement.

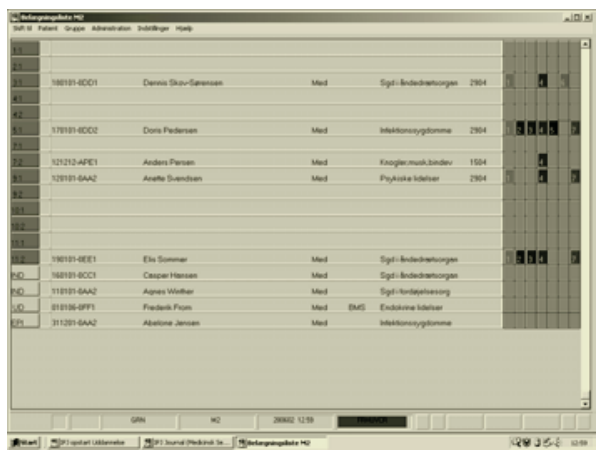

Fig. 1. Electronic patient record: List of patients on the ward.

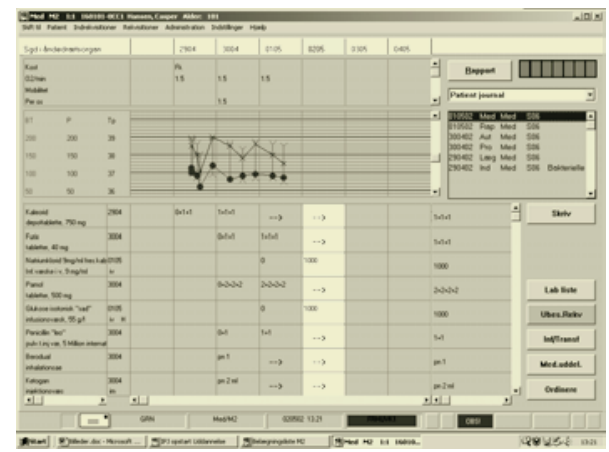

Fig. 2. Electronic patient record: detailed information about a specific patient. 


\subsection{Findings}

The evaluation of the electronic patient record identified a substantial amount of usability problems. The total was 75 usability problems distributed on 9 critical, 39 serious and 27 cosmetic problems [8]. These 75 usability problems relate to various concerns of human-computer interaction, e.g. finding information, storing information, complexity of screen layouts. On the other hand, the usability test also identified a number of strengths in the interface of the electronic patient record, e.g. easy registration of values, well integrated in the work activities, good overview of the patients in the ward. The following three themes reflect more abstract concerns of the usability of the electronic patient record.

Mobility. During the usability evaluation and in the interview afterwards, most nurses would stress concerns about being mobile while working with the system. Meeting this challenge, the use of laptop computers rather than desktop workstations had been suggested and discussed at the hospital. However, most of the nurses stated that they would find it impossible or unfeasible to carry a laptop computer around the ward every time they were to conduct work tasks away from their office. One problem was the size of the laptop, as they would also have to carry other instruments.

Complexity. Another overall concern or problem was complexity and fragmentation of information. Most nurses found it difficult to locate the necessary patient information as they were solving tasks. This sometimes led to inadequate or incomplete task completion. Hence, the nurses would be insecure whether they had found the right information and whether they had succeeded in finding all relevant information.

Work Relation. Most nurses experienced problems in the laboratory due to the fact that they had difficulties in relating the test-tasks to carrying out of real work activities. The problem was that they would typically use different kinds of information in the "real life" to determine how to solve a problem, e.g. the visible condition of a patient. Another concern related to the fact that the system only partially reflected the current work task, making it difficult to the test subjects to find or store information.

\section{MobileWARD}

Motivated by the findings from our evaluation of the stationary electronic patient record system, a field study was conducted into the work activities at the hospital of Frederikshavn related to the use of electronic patient records in practice. In concert with the findings from the usability evaluation, this suggested a design solution that 1) supported the highly mobile work activities of nurses by being handheld, 2) reduced complexity by adapting to its context and 3) eliminated double registering of information (first written down on paper and then entered into the PC later) by being integrated with the existing patient record. While facilitating access to patient information at the 'point of care' is not a new idea [1, 10, 15], adapting information and functionality in a mobile EPR system to its context is a novel approach to improving the usability of such systems, which has not yet been investigated thoroughly. On the basis of the findings from the usability evaluation and subsequent field study, an experimental prototype of a handheld context-aware EPR system for supporting the 
morning procedure, was designed and implemented [6]. The system is described briefly below.

\subsection{Architecture}

MobileWARD is designed for a Compaq iPAQ 3630 running the Microsoft ${ }^{\circledR}$ PocketPC operating system. The system uses a Wireless Local Area Network (wLAN) for network communication. The system was implemented in Microsoft embedded Visual Basic 3.0. For the experimental prototype of MobileWARD, context awareness was simulated by means of a "context control center" application. The control center runs on a separate iPAQ connected to the wireless network. Through this application, an operator can trigger "context events" in MobileWARD, simulating that the user has entered a specific room, scanned the barcode on a specific patient etc. This approach was chosen to facilitate early evaluation of the experimental design solution without having to worry about the technical challenges of context sensing. In later versions of the system, real sensing of the environment will be implemented where promising. For discussions on how to sense environments see for example [3] or [14].

\subsection{Interface Design}

MobileWARD is designed to support work tasks during morning procedure at the hospital ward. The design is based on two basic concepts. First, the system is designed to reflect the context of the user in the sense that it is able to sense and react to a number of changes in the environment. Secondly, as the use of a pen for typing in information would sometimes be inappropriate because the nurses would often use the system while being mobile or engaged in other activities, the interface design incorporates a visual layout with large-scale buttons that enables finger-based interaction through the touch-screen of the iPAQ.

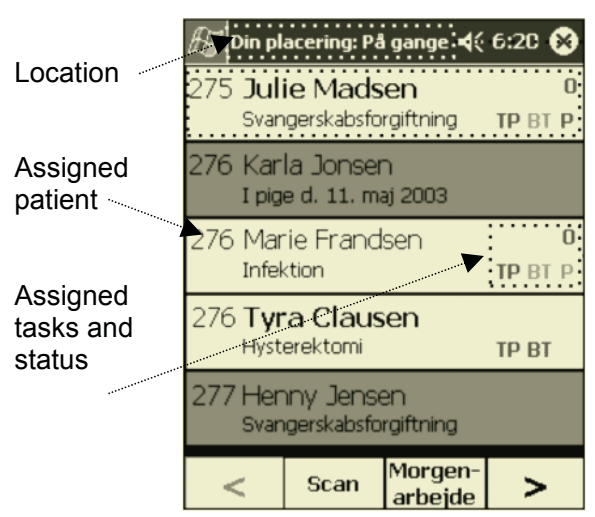

Fig. 3. MobileWARD. Patient lists

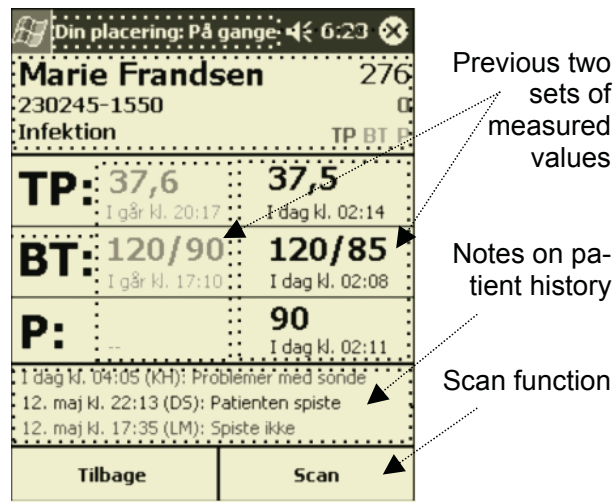

Fig. 4. MobileWARD. Patient information 
MobileWARD is context-aware in the sense that the system recognises the location of the nurse and presents information and functionality accordingly. Before visiting assigned patients, the nurses often want to get an overview of the specific information about each patient, e.g. previous measured values. This typical takes place at the nurse's office or in the corridor. The windows related to these locations are shown in figure 3 and 4 . When in the corridor, the system by default displays all the patients on the wards. The patient list is ordered by ward number. Patients assigned for morning procedure are shown with a white background and the names of patients assigned to the nurse using the system are boldfaced (e.g. "Julie Madsen" and "Tyra Clausen" on figure 3). At the top of all windows, the nurse can see their current physical location as interpreted by the system. In the example on figure 3, the nurse is in the corridor ("på gangen"). For each patient, MobileWARD provides information about previous tasks, upcoming tasks and upcoming operations. The indicators TP (temperature), BT (blood pressure) and P (pulse) show the measurements that the nurse has to perform. The indicators are either presented with red text (value still to be measured) or green text (value already measured). Above the three indicators, an " $\mathrm{O}$ " indicates an upcoming operation (within 24 hours), which usually requires that the patient should fast and be prepared for operation. If the nurse wants to view data about a specific patient, she can click on one of the patients on the list. This will open the window shown on figure 4, displaying the name and personal identification number of the patient, the previous two sets of temperature, blood pressure, and pulse measurements taken as well as written notes regarding the treatment of the patient. This window is accessible at any time and location. Thus the nurse can choose to look up more specific details about each patient while located in the corridor or in the office. In order to enter new data into the system, the nurse has to scan the barcode identification tag on the patient's wristband using the "scan" function in the bottom of the screen. This is described further below.
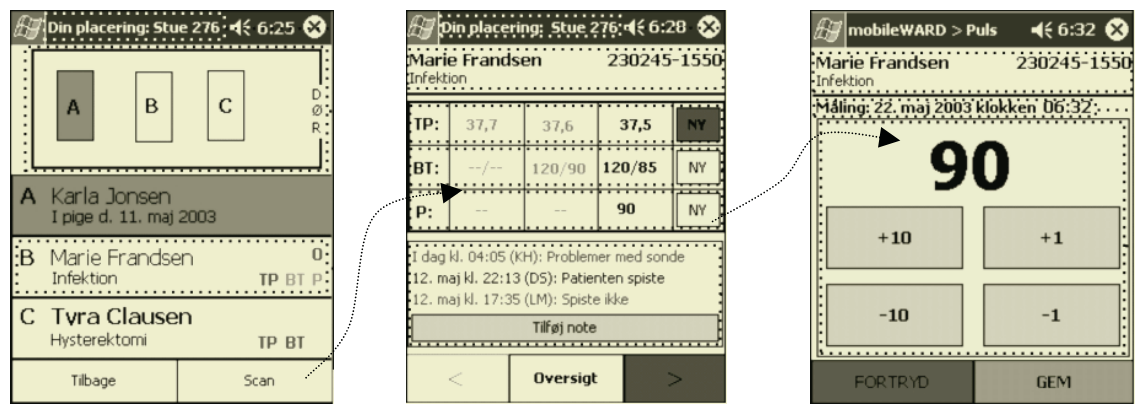

Fig. 5. Windows displayed in the ward in relation to the tasks of measuring temperature, blood pressure, and pulse.

The aim of this design is to provide the nurse with information that helps her plan the scheduled morning procedure. The system presents information and functionality adapted to the location of the nurse and the time of the day. Furthermore, the system knows the status of each patient and represents already measured values and values yet to be measured by simple colour codes. 
When the nurse enters a ward, the system automatically displays a different set of information. This is illustrated in figure 5. At the top of the screen, the nurse can see her physical location as interpreted by the system (e.g. ward 276). Below this, information about the patients on the current ward is presented, resembling the information available on the patient list displayed in the corridor, with the addition of a graphical representation of the physical location of the patient's respective beds. In this way, MobileWARD aims at presenting only relevant information to the nurse, e.g. by excluding patients from other wards. Like in the corridor, data about the patients is available by clicking on their names (or on their bed-icon). At the bottom of the screen, the nurse can activate the barcode scanner ("scan") used to identify a patient prior to entering data into the system. After having scanned a patient, the nurse can type in measured values (figure 5, center). This window shows previous measurements of values and provides functionality for typing in new values. By clicking the new value button ("ny"), the system displays a window for entering new values (figure 5, right). Below the personal information (name and personal identification number), date and time is shown. In the gray box, the nurse can input the measured value by editing the shown value. This is done by pressing the large sized buttons on the screen with a finger. The number shown by default is the latest measurement. The reason for this is that the latest measure is most likely to be close to the newest one. If there is a huge difference, this is clearly signaled to the nurse, as she will have to perform more button presses than usual, providing an implicit opportunity to see whether, e.g. the temperature for a given patient is rising or falling. The Save button stores the value in the database along with the date, time, and user identification. Furthermore, the system updates the status of the task as having been carried out.

\subsection{Usability Evaluation of MobileWARD}

We designed and conducted two different usability evaluations of the mobile EPR system MobileWARD. The evaluations were similar as they involved trained, registered nurses as test subjects, who should conduct standard morning work routines. However, they were different in their setting and data collection. The participating subjects were between 27 and 54 years old and they had diverse experiences with nursing. All of them were novices with the use of handheld computers.

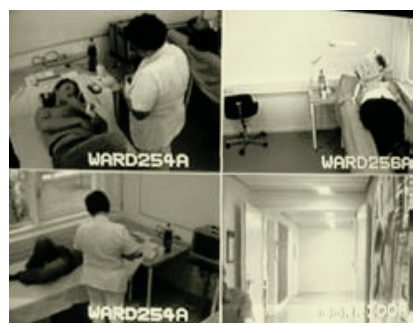

Fig. 6. Laboratory evaluation

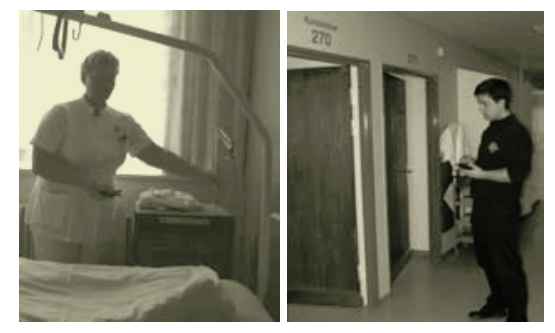

Fig. 7. Evaluation at the hospital

The first evaluation took place at the usability laboratory at Aalborg University. The idea of this evaluation was to evaluate the mobile system in an environment 
where we could closely monitor all actions and situations (figure 6). Three test subjects participated in the study. They were all given a series of tasks to perform while using the system, and were asked to think-aloud. Three students acted as patients for the evaluation. One researcher acted as test monitor while another controlled the video equipment in the control room. The second evaluation took place at the Hospital of Frederikshavn. The field evaluation focused on using the system in a realistic environment and took place during evening procedure with real patients. Prior the evaluation, data on the patients at the ward was entered into the system. The use of the system was not controlled by task assignments but the nurses were asked to use it for registering the data they would be measured during the evening procedure (figure 7).

\subsection{Findings}

In a preliminary analysis of the evaluations, we have identified a total of 16 usability problems. These problems can be divided into three categories, concerning interaction, mobility, and context-awareness. First, interaction problems concerned nurses having problems with interacting with the system or understanding the interface. E.g. one nurse did not know the semantics of the keyboard for typing in textual notes about patients. Specifically, she felt insecure about the buttons "Tab", "Caps" and "Shift" as she would expect them to be "tablets", "capsules" and "shift medication". Secondly, other problems concerned aspects of mobility and working conditions, e.g. one nurse was concerned about putting the mobile device in her pocket. She was afraid that she would accidentally click some buttons while walking and she stated that it would be impossible to carry the device in the hand at all times. Another problem related to mobility and working conditions was the fact that one nurse feared that the device could spread bacteria from patient to patient. Thus, she did not want to place the device on the patient's bedside table or on the bed. Thirdly, some problems concerned aspects of context-awareness, e.g. the automatic adaptation of the interface to location. One of the nurses was reading information on the device while walking into the ward and got confused when the interface suddenly changed its content without notice or without her trigger anything.

\section{Discussion}

Through a usability evaluation of a traditional electronic patient record, we identified a number of important issues to consider when supporting work tasks in health care namely mobility, complexity, and work tasks.

Mobility. We found that mobility issues were important in professional nursing work activities. Nurses would normally find themselves visiting patients in different physical locations and they often require several kinds of information for more work tasks. We attempted to support mobility through a relatively small, handheld device that could be carried around by the nurses (potentially in their pockets) while visiting patients or conducting other work tasks. The idea of having a mobile device was 
adapted by all nurses in our evaluation. However, the nurses would switch between reading/storing information on the device and conducting work tasks without the device e.g. taking measurements from patients. Hence, having the device in the hands all the time would be impossible and thus, they would occasionally need to put away or lay down the device. Some of them requested functionalities that would allow them to lock the screen.

Complexity. Another important issue identified in our first evaluation was problems related to complexity and fragmentation of information. Most nurses experienced problems in locating relevant and adequate information in the traditional electronic patient record. This was mainly due to extensive amount of different types of information on each screen (figure 2). As a result, they occasionally failed to notice relevant or even critical information on e.g. patients and scheduled operations. On the mobile device, we aimed at presenting much less information at a time to address this problem. The nurses found no severe complexity problems while using the mobile device. However, they would occasionally request more information than could be fitted into the screen at one time. Exploiting context-awareness, e.g. physical location of users and patients, furthermore reduced the complexity of information. Thus, we were, for example, able to present information about patients close by only.

Work Relation. From the usability evaluation and field studies, we found that nurses would typically require very specific information based on current work tasks and activities. The traditional electronic patient record did not fully support this but presented too much, too little, or too fragmented information. In the mobile EPR prototype, we again utilized context-awareness in different ways as a mean for determining the work task of the nurses. However, this also introduced some pitfalls as nurses would sometimes miss reminders presented on the screen because they their focus was engaged elsewhere. Furthermore, some nurses became confused or even annoyed by the automatic adaptation of information on the screen to their physical location. Thus, the idea of context-awareness would not always support the nurses' work activities. On the contrary, it was sometimes seen as an obstacle to interacting with the system.

\section{Conclusions}

Supporting work activities in healthcare has been found to be a highly complex and challenging task [2]. We have conducted a study with two aims. First, we identified important issues in supporting work tasks in healthcare through the evaluation of a traditional desktop-based electronic patient record. Secondly, a mobile electronic patient record prototype that would address the identified challenges from the first evaluation was designed, implemented, and evaluated. The mobile prototype utilized context-awareness as a key means for supporting the nurses' use of the EPR system. Our results indicate that work tasks can be supported through a mobile, handheld device, but that healthcare work is highly complex which needs further investigations. Also, our findings have challenged the prevalent view of context-awareness as a universally useful paradigm for interacting with mobile computer systems. 


\section{Acknowledgements}

The authors thank all test subjects who participated in the usability evaluations and the Hospital of Frederikshavn for kind collaboration throughout the project. The usability evaluations were conducted in collaboration with Jan Stage, Benedikte Skibsted Als and Rune Thaarup Høegh. MobileWARD was designed by Rune Thaarup Høegh, Karsten Kryger Hansen and Søren Lauritsen.

\section{References}

1. Arshad, U., Mascolo, C. And Mellor, M. (2003) Exploiting Mobile Computing in Healthcare. In Demo Session of the 3rd International Workshop on Smart Appliances, ICDCS03

2. Bardram, J., Kjær, T. A. K., and Nielsen, C. (2003) Supporting Local Mobility in Healthcare by Application Roaming among Heterogeneous Devices. In Proceedings of Mobile HCI'03, Springer-Verlag, LNCS

3. Bohnenberger, T., Jameson, A., Krüger, A., and Butz, A. (2002) Location-Aware Shopping Assiatnce: Evaluation of a Decision-Theoretic Approach. In Proceedings of Mobile HCI'02, Springer-Verlag, LNCS, pp. 155-169

4. Brinck, T. And York, G. (1998) User Interfaces for Computer-Based Patient Records. In Proceedings of CHI'98, ACM

5. Grimson, J. And Grimson, W. (2000) The SI Challenge in Health Care. Communications of the ACM 43(6), pp. 49-55

6. Hansen, K. K. Høegh, R. T. and Lauritsen, S. (2003) Making Context-Awareness (at) Work. June, 2003, Aalborg University

7. Kaplan, S. M. and Fitzpatrick, G. (1997) Designing Support for Remote Intensive-Care Telehealth using the Locales Framework. In Proceedings of DIS'97, ACM

8. Kjeldskov, J., Skov, M. B. and Stage, J. (2002) Usability Evaluation of IBM IPJ 2.3 Electronic Patient Record (in Danish), June, 2002, Aalborg University

9. Molich, R. (2000) Usable Web Design (In Danish). Ingeniøren | bøger

10. Morton, S. and Bukhres, O. (1997) Utilizing mobile computing in the Wishard Memorial Hospital ambulatory service. In Proceedings of SAC'97, ACM

11. Nielsen, J. (1993) Usability Engineering. Morgan Kaufmann

12. Rindfleish, T. C (1997) Privacy, Information Technology, and Health Care. Communications of the ACM 40(8), pp. 93-100

13. Rubin, J. (1994) Handbook of Usability Testing. Wiley

14. Schilit, B. N. and Theimer, M. M. (1994) Disseminating Active Map information to Mobile Hosts. In IEEE Network, Vol. 8(5), pp. 22-32

15. Urban, M. and Kunath, M (2002) Design, Deployment and Evaluation of a Clinical Information System which uses Mobile Computers and Workstations. In Proceedings of the 2nd conference on Mobiles Computing in der Medizin, Heidelberg 